\title{
MIGRATIONS ET FLUX MONÉTAIRES : QUAND CEUX QUI RESTENT FINANCENT CELUI QUI PART
}

\section{Hugo Bréant}

Presses de Sciences Po (P.F.N.S.P.) | « Autrepart »

2013/4 Nº 67-68 | pages 31 à 52

ISSN 1278-3986

ISBN 9782724633108

Article disponible en ligne à l'adresse :

http://www.cairn.info/revue-autrepart-2013-4-page-31.htm

\section{!Pour citer cet article :}

Hugo Bréant, « Migrations et flux monétaires : quand ceux qui restent financent celui qui part », Autrepart 2013/4 (N 67-68), p. 31-52.

DOI 10.3917/autr.067.0031

Distribution électronique Cairn.info pour Presses de Sciences Po (P.F.N.S.P.).

(C) Presses de Sciences Po (P.F.N.S.P.). Tous droits réservés pour tous pays.

La reproduction ou représentation de cet article, notamment par photocopie, n'est autorisée que dans les limites des conditions générales d'utilisation du site ou, le cas échéant, des conditions générales de la licence souscrite par votre établissement. Toute autre reproduction ou représentation, en tout ou partie, sous quelque forme et de quelque manière que ce soit, est interdite sauf accord préalable et écrit de l'éditeur, en dehors des cas prévus par la législation en vigueur en France. Il est précisé que son stockage dans une base de données est également interdit. 


\title{
Migrations et flux monétaires : quand ceux qui restent financent celui qui part
}

\author{
Hugo Bréant*
}

Que les migrants soient décrits comme des aventuriers, des commerçants transfrontaliers ou des courtiers en développement [Schmitz, 2008], les mobilités humaines et les flux d'argent semblent toujours consubstantiels. Rien d'étonnant, donc, à voir ces deux sujets très tôt étudiés ensemble. Depuis les années 1970 en effet, la sociologie et l'anthropologie des mobilités s'intéressent à la circulation de l'argent liée aux migrations, et notamment à cette pratique sociale courante qu'est l'envoi de fonds de l'immigré vers son pays d'origine. Bien souvent, c'est une sorte de nature de la condition d'immigré qui est mise en avant. Cette nature consiste à donner de l'argent à ses proches, et par là à attester de son attachement et de la force des liens familiaux transnationaux. En 1991, par exemple, Catherine Quiminal publie Gens d'ici, gens d'ailleurs, un livre consacré aux migrations maliennes soninkés en France. Dans un chapitre intitulé «Le Sens de l'argent», elle résume cette situation: «Tandis que le village s'allie avec la famille pour multiplier les demandes d'argent, les salaires des fils, caristes, éboueurs, manœuvres, balayeurs, blanchisseurs, ne s'éloignent guère du SMIG [...] pourtant, quand on est en France, il faut économiser pour le village, la famille, épargner pour l'avenir » [1991, p. 112]. Cette tendance observée par les chercheurs est par ailleurs relayée par les médias, insistant ainsi sur le sens du sacrifice de l'émigré et diffusant une vision à la fois misérabiliste et héroïsante de la migration : À 40 ans, Moriba a passé plus de la moitié de sa vie en France. Chaque mois, il envoie de l'argent à sa famille au Mali. Électricien dans une grosse entreprise privée, Moriba touche 1600 euros par mois. Avec le loyer de 300 euros pour sa chambre dans un foyer de travailleurs, l'argent envoyé à ses proches représente le deuxième plus gros poste de dépense de son modeste budget. Dans son village de la région de Kayes (ouest du Mali), il subvient aux besoins de sa femme et de ses quatre enfants. Il aide ses vieux parents, ses frères et sœurs et leurs propres enfants.

\footnotetext{
* Doctorant en science politique, Centre européen de sociologie et de science politique (CESSP), université Paris 1 - Panthéon Sorbonne et Attaché temporaire d'enseignement et de recherche (ATER) en science politique à l'Institut de recherche interdisciplinaire en sciences sociales (IRISSO), université Paris Dauphine.
} 
Moriba envoie 250 euros par mois, auxquels s'ajoutent des aides ponctuelles (problèmes de santé, mariage). Par ailleurs, la très grande majorité des Africains immigrés se regroupent au sein d'associations villageoises qui organisent les collectes. Les sommes alimentent les fameuses «tontines », système d'épargne rotative, ou bien permettent d'investir au village, pour la construction d'un puits ou d'une école. « La dernière fois que j'ai été sollicité, raconte Moriba, j'ai donné 700 euros pour un projet d'adduction d'eau. Il peut y avoir deux ou trois cotisations par an. » [Gorce, 2008]

Ce phénomène a également suscité l'intérêt du champ politique, et chercheurs et acteurs politiques ont parfois travaillé de manière conjointe. À cet égard, la recherche par sondages engagée par l'équipe du groupe d'étude des migrations internationales de la Fondation nationale des sciences politiques, sous la direction de Jean-Pierre Garson et Georges Tapinos, publiée en 1981 sous le titre L'Argent des immigrés, est emblématique. L'initiative avait été lancée par une mission interministérielle des ministères du Travail et des Finances, pour évaluer «les effets de l'immigration sur la balance des paiements » et « identifier les variables explicatives du comportement de transfert »[Garson, Tapinos, 1981]. Les frontières entre champs académique, politique et associatif peuvent donc parfois être très poreuses [Dedieu, 2012, en particulier le chapitre 3, «Les développeurs »].

Cette façon commune de penser les migrations internationales par le prisme de leur dimension monétaire et comptable fait écho de manière plus générale à une conception des migrations en termes de coûts et de profits. D'après Abdelmalek Sayad, «L'immigration n'a de sens, et n'est intelligible pour l'entendement politique, qu'à la condition qu'elle soit source de "profits" ou, pour le moins, que les "coûts" qu'on lui impute n'excèdent pas les "profits" qu'elle peut procurer. » [1999, p. 118] Dès lors, l'argent des migrations devient nécessairement un élément de réflexion important. Mais si la participation à l'activité économique du pays d'accueil, qui a pour corollaire l'envoi d'argent dans le pays d'origine, est présentée comme une réalité sociale qui peut profiter à toutes les parties en présence, il est intéressant de noter que cet argent, lié à des expériences migratoires individuelles, semble circuler toujours dans le même sens. L'argent part du Nord pour arriver au Sud, le migrant devenant à la fois celui qui produit le revenu, qui l'économise, le partage, le transfère ou le distribue.

Pourtant, qu'elle s'effectue selon des modalités légales ou non, l'émigration nécessite d'avoir des ressources financières, et partir de son pays peut nécessiter de contracter des emprunts. On peut alors se demander si l'argent circule inévitablement de manière unidirectionnelle et s'il n'y a pas de flux monétaires à contresens.

En se basant sur un double terrain d'enquête portant sur les migrations togolaises et comoriennes en France ${ }^{1}$, cet article cherche à se distancier du double

1. Ma thèse porte sur les trajectoires d'émigrés et interroge le poids de la différenciation sociale dans les profils migratoires individuels et collectifs. Les cas togolais et comoriens ont été très peu étudiés. Les 
mouvement qui consiste, d'une part, à naturaliser la pratique des envois de fonds, et d'autre part, à rendre poreuse la frontière entre problématiques politiques et problématisations académiques autour de la question de l'argent en migration. L'objectif n'est pas ici de donner un cadre d'analyse précis pour l'étude de la circulation monétaire en migration depuis le Sud jusqu'au Nord ${ }^{2}$, mais d'ébaucher quelques pistes pour essayer de comprendre pourquoi cette circulation monétaire, qui va de la famille restée dans le pays d'origine à l'émigré arrivé dans le pays d'accueil, n'est jamais mise en lumière, et ce que son étude peut permettre de révéler dans les recherches sur les migrations internationales. Dans un premier temps, il s'agira de constater à quel point cette autre réalité sociale a été placée dans l'ombre, hors de l'agenda sur les migrations internationales. Quelques pistes d'explication à cette faible mise en visibilité seront avancées. Par la suite, l'article aura surtout pour ambition de mettre en avant la circulation du Sud au Nord, d'en donner quelques contours sociologiques et surtout de montrer que ces envois peu visibles ont pourtant un réel intérêt, en ce qu'ils peuvent stimuler des réflexions plus générales sur les profils migratoires eux-mêmes.

\section{Du Nord au Sud, I'immigré comme pourvoyeur d'argent}

Dans une interview donnée à Radio France Internationale, un conseiller de l'Agence française de développement (AFD) expliquait que dès 1975 «L'AFD, qui s'appelait alors Caisse centrale de coopération économique, avait mis en place des programmes d'appui au développement des pays d'origine [...] ensuite, on s'est intéressé aux transferts pour arriver à en canaliser une partie vers des investissements productifs ou sociaux. » [Marsaud, 2006] La préoccupation des institutions nationales et internationales pour l'argent des migrations n'est donc pas nouvelle, mais elle prend de plus en plus de poids dans les années 2000, jusqu'à devenir un objet central et un véritable passage obligé [Kapur, 2004].

Cette redécouverte des envois de fonds ${ }^{3}$ coïncide avec l'encouragement par la Banque mondiale et le Fonds monétaire international (FMI) d'une politique de

différences entre ces deux cas (deux histoires migratoires décalées dans le temps, deux migrations politiquement construites en France comme plus ou moins désirables, deux profils migratoires opposés, l'un étant jugé plus individualiste et élitiste, l'autre plus communautaire et précaire) permettent d'analyser de manière contrastée les conditions familiales, sociales et politiques des mobilités. J'ai mené une enquête multisituée et réalisé des entretiens sociologiques avec des personnes qui souhaitaient partir, avec des proches d'émigrés, avec des émigrés en France ou lors de retours en vacances et avec des émigrés revenus s'installer dans leur pays d'origine. Les entretiens réalisés ont pris la forme de récits de vie que j'ai essayé, dans la mesure du possible, de transformer en «entretiens ethnographiques », c'est-à-dire en entretiens « approfondis » et «enchâssés dans l'enquête de terrain » [Beaud, 1996, p. 234]. Mes différents terrains de recherches (au Togo en 2010, aux Comores en 2012 et en France entre 2010 et 2013) m'ont à ce jour permis de réaliser 70 entretiens sociologiques dans le cas togolais et 127 entretiens dans le cas comorien.

2. La circulation monétaire ne fait pas partie des préoccupations centrales de mes recherches, mais comme beaucoup de chercheurs qui travaillent sur les migrations, j'y ai été très souvent confronté lors de mes enquêtes de terrain, ce qui a suscité un certain nombre d'interrogations que je livre ici.

3. "In the past few years, there has been a remarkable renaissance in the interest in remittances. This interest has undoubtedly been triggered by a striking increase in remittance flows : after years of relative neglect, they have been rediscovered as a potential source of development finance." [De Haas, 2007, p. 1]. 
coopération et de développement libérée des structures étatiques et fondée sur les initiatives privées [Freud, 2009; Hibou, 1998]. Ainsi, l'efficacité de l'aide publique au développement a été remise en cause [Carbonnier, 2010] alors que le poids des migrants comme acteurs individuels du développement a été valorisé ${ }^{4}$.

Plusieurs organisations internationales participent à cette mise sur agenda de la question des transferts de fonds, mais la Banque mondiale est devenue la plus puissante initiatrice de cette accélération depuis le tournant des années 2000. Il suffit de visiter son site Internet pour se rendre compte que c'est par le biais de l'argent que les migrations internationales y sont abordées. En effet, la section s'intitule « Migrations et envois de fonds », ce qui suggère que les deux sont, pour elle, inextricablement liés. Régulièrement, l'institution publie une note d'informations sur ce thème, à laquelle s'ajoutent des données disponibles en ligne, la publication annuelle d'un Factbook et surtout, la diffusion de nombreux rapports. En mai 2012, grâce aux fonds de l'Agence suisse pour la coopération et le développement (SDC), la Banque mondiale a mis en place le Global Knowledge Partnership on Migration and Development (KNOMAD), un groupe multidisciplinaire destiné à améliorer et mettre en commun les connaissances liées au rôle des migrations internationales dans le développement. Mais l'orientation de la Banque mondiale est claire. Malgré ses appels répétés à un approfondissement des connaissances, le discours est normatif : «Les quelque 30 millions d'Africains qui vivent aujourd'hui à l'étranger constituent une ressource vitale pour le continent, mais les responsables africains doivent encore faire plus pour tirer tous les avantages économiques de ce phénomène de migration. » Voilà ce que l'on pouvait lire en mars 2011 sur le site français de la Banque mondiale [2011a]. Il est désormais naturel de penser que les 62 millions de migrants venus des pays du Sud vers des pays du Nord contribuent, par leurs envois de fonds, au développement de leur pays d'origine et au recul de la pauvreté. Ils sont devenus, sans que ce fait paraisse questionné, des acteurs incontestables du développement. Deux autres institutions américaines ont participé à l'initiative de la Banque mondiale : la Banque interaméricaine de développement (BID), qui a lancé plusieurs études et projets depuis 2000, par le biais du Fonds d'investissement multilatéral (FOMIN), et l'Agence des États-Unis pour le développement international (USAID), qui finance de multiples projets liés aux envois de fonds ${ }^{5}$.

Bien entendu, ces organisations ne sont pas les seules à s'intéresser à ces questions et à les transformer progressivement en thématique incontournable. Parmi les autres organisations internationales, l'Organisation internationale des

4. «Les travaux menés notamment au sein du G8 - un groupe d'experts G8 a été créé à la suite des conclusions du sommet du G8 de juillet 2008 - et du Groupe pilote sur les financements innovants du développement - témoignent de la volonté des États de faciliter ces transferts d'argent privés complémentaires à l'aide publique au développement, de baisser leur coût et de développer des incitations en faveur de leur utilisation à des fins d'investissement productif. » [ministère des Affaires étrangères, 2008].

5. Comme le Diaspora Networks Alliance (DNA), dont le but est de cibler, d'étudier et de développer les ressources qui permettent aux migrants de participer au développement, ou le Migration and Development Fund (MDF), qui vise à financer des initiatives locales et à optimiser les transferts. 
migrations (OIM) et l'Organisation des Nations unies (ONU) ${ }^{6}$ ont contribué à intégrer ce sujet dans les deux Dialogues de haut niveau des Nations Unies sur les migrations internationales et le développement (en 2006 et en 2013). Enfin, l'Organisation de coopération et de développement économiques (OCDE) et le Programme des Nations Unies pour le développement (PNUD) ont régulièrement publié des rapports sur le sujet [SOPEMI, 2006 ; Klugman, 2009].

La mise sur agenda des transferts de fonds des migrants à l'échelle internationale s'accompagne d'une forte activité à l'échelle régionale. En Afrique par exemple, où les coûts de transferts sont les plus élevés, la Banque africaine de développement (BAD) a activement participé à ce mouvement général. Depuis une étude conduite en 2007 dans quatre pays (Comores, Mali, Maroc et Sénégal) [BAD, 2008], la BAD a multiplié les partenariats. En 2009, avec l'aide du Fonds international de développement agricole (FIDA) et du ministère de l'Immigration français, elle a créé le Fonds fiduciaire sur les transferts de fonds des migrants et a lancé l'Initiative migrations et développement, chargée de produire des rapports et de soutenir des projets dans le but de mobiliser, de renforcer, d'encadrer, de pérenniser et de rendre productifs ces transferts. Malgré une forte activité de production de rapports, notamment en partenariat avec les équipes de la Banque mondiale [Ratha, 2011], l'institution africaine cherche avant tout à créer concrètement de nouveaux produits financiers et, comme elle l'indique sur son site, à « promouvoir les alliances et le réseautage stratégique ». En juin 2013, un Forum sur les transferts de fonds en Afrique a par exemple eu lieu dans le Palais de l'Assemblée nationale, à Praia, au Cap-Vert.

C'est cet activisme largement partagé à toutes les échelles qui a mené à la mise en place en 2004, après le sommet du G8 de Sea Island, à la création d'une initiative globale, le Global Remittance Initiative (GRI), qui visait à encourager les transferts formels et la baisse de leurs coûts par l'accroissement de la concurrence $^{7}$, et plus généralement cherchait une meilleure coordination entre organisations internationales, institutions financières locales, prestataires de services financiers et migrants [Coiffard, 2012].

Il apparaît que les transferts de fonds des migrants sont devenus un sujet à côté duquel plus aucun sommet lié aux migrations ne peut passer. Mais surtout, ce thème s'accompagne désormais d'un discours relativement normatif dans lequel on laisse peu de place aux débats sur les conséquences effectives de ces transferts, mais où l'on met en avant le fait qu'ils ne paraissent pas assez productifs, qu'ils

\footnotetext{
6. Notamment par le biais de son département des Affaires sociales et économiques et de la Conférence des Nations unies sur le commerce et le développement (CNUCED).

7. En 2011, la Banque mondiale crée, avec l'appui du projet d'Institut africain pour les envois de fonds des migrants (AIR), la base de données «Send Money Africa ». Sur le site, on peut lire cet appel clair à un accroissement de la concurrence : «Les tarifs des envois de fonds sont encore plus élevés entre les nations africaines. C'est en Afrique du Sud, en Tanzanie et au Ghana qu'ils sont les moins avantageux : ils se montent respectivement à $20,7 \%, 19,7 \%$ et $19 \%$ en moyenne, en raison de divers facteurs, dont la faible concurrence qui prévaut sur le marché des transferts de fonds internationaux. » [Banque mondiale, 2013].
} 
sont trop coûteux, surtout en Afrique, et qu'il faut donc essayer de les encourager tout en les canalisant.

La mise en scène statistique de l'importance croissante de ces envois d'argent, de leur capacité à résister aux crises économiques et de leurs impacts locaux reste d'ailleurs une stratégie courante des organisations internationales pour justifier le rôle de ces flux dans le développement des pays du Sud [Bréant, 2012].

L'argent des diasporas est au cœur des politiques internationales depuis plus de dix ans. Toutefois, cet argent est toujours celui des immigrés dans les pays d'accueil à destination de leur famille dans les pays d'origine. Les envois de fonds apparaissent à sens unique. En effet, la profusion de données institutionnelles sur les flux du Nord au Sud contraste avec l'absence quasi totale de données sur l'argent qui circule au sein du Sud ou du Sud au Nord dans le cadre de migrations régionales ou internationales.

Dans un contexte de recomposition des relations internationales, plusieurs chercheurs commencent à se pencher par exemple sur les pays du Sud qui financent la dette des pays du Nord [Gurtner, 2007]. Mais rien n'est encore fait sur la question migratoire ${ }^{8}$, même si les migrations de citoyens du Nord vers des pays du Sud commencent à émerger médiatiquement et annoncent peut-être de nouvelles thématiques de recherches [Gatinois, 2012 ; Filipe, João Babo, 2013].

Les organisations internationales reconnaissent d'ailleurs elles-mêmes ce manque de données puisque l'Observatoire des pays d'Afrique, Caraïbes et Pacifique (ACP) sur les migrations et l'Initiative conjointe Commission européenne-Nations unies pour la migration et le développement (ICMD) ont lancé un partenariat durant l'été 2012 pour « promouvoir une attention accrue aux migrations et développement Sud-Sud » [Observatoire, 2012].

\section{Difficultés et intérêts à rendre visibles certains flux monétaires}

On peut désormais essayer d'ébaucher des pistes d'explications face à ce constat qui veut que les transferts d'argent en migration soient devenus un thème central des programmes des organisations internationales, sans que la circulation soit pensée autrement que partant du Nord pour arriver au Sud.

L'argument de la difficulté technique à connaître ces flux peut bien sûr être la première cause de cette invisibilité. Lorsque les organisations internationales tentent de chiffrer les transferts de fonds, elles s'appuient sur les données de l'argent envoyé via des sociétés de transferts ou par le biais de la bi-bancarisation, c'està-dire de l'envoi d'argent via des virements sur des comptes bancaires communs ou partenaires. Elles tentent également de chiffrer les transferts informels. Lors

8. Une simple requête effectuée en mars 2013 dans le moteur de recherches Google permet de constater cet écart colossal : 71000 résultats pour l'expression «argent envoyé par les migrants », 2 résultats pour « argent reçu par les migrants » et aucun résultat pour «argent envoyé aux migrants ». 
d'un entretien avec Younoussa Imani, enseignant à l'université des Comores qui a soutenu en 2011 une thèse de sciences économiques à l'université de Bordeaux IV sur les transferts de fonds aux Comores, celui-ci expliquait que, « à $80 \%$, les gens utilisent la méthode traditionnelle ». Autrement dit, l'argent est confié de la main à la main au sein de la famille, ou une valise est confiée à un compatriote pour qu'il la transmette à Paris ou à Moroni, selon le sens du voyage et de la circulation de l'argent ${ }^{9}$. Cette méfiance à l'égard des institutions bancaires et/ou ce refus de payer pour un service qui peut être réalisé de manière informelle se retrouve dans d'autres pays, comme Haïti [Laëthier, 2011, p. 168]. Il apparaît difficile de mesurer réellement ces pratiques informelles. Le 2 août 2012 par exemple, l'Observatoire des coûts d'envoi d'argent à l'étranger explique, en s'appuyant sur l'exemple comorien, que « D'après la Banque centrale comorienne, les transferts informels seraient estimés à $65 \%$ en 2010 contre $71 \%$ en 2009. Cette baisse s'explique en partie par le rôle accru des sociétés de transfert d'argent (STA) dans le pays. » [AFD, 2012] Mais les problèmes techniques ne suffisent pas à expliquer l'absence de données sur ces flux. En effet, le fait de ne pas disposer de données fiables n'a jamais empêché les organisations internationales d'écrire des rapports sur les transferts de fonds du Nord au Sud.

Si le discours s'avère faiblement critique et unanimement enthousiaste autour des transferts de fonds du Nord au Sud, c'est peut-être aussi parce que ceux-ci mettent en jeu des intérêts financiers très importants, notamment pour les sociétés américaines de transferts d'argent comme Money Gram, Ria, Coinstar ou Western Union. Le chiffre d'affaires de cette dernière s'élevait à 5,2 milliards de dollars en 2010 [Mboungou, 2012]. Ces transferts de fonds intéressent parce qu'ils peuvent être un instrument utilisé dans le cadre de politiques de développement, mais aussi parce qu'ils représentent un vaste marché qui est l'objet de nombreuses attentions ${ }^{10}$. En 2006, Money Gram et Ria ont obtenu l'agrément du Comité des établissements de crédit et commencé à concurrencer en France l'entreprise Western Union qui était jusque-là très majoritaire, après avoir obtenu en 1994 un partenariat exclusif avec La Poste. En octobre 2006, Charles Milhaud, alors président du Groupe Caisse d'épargne, remettait au ministère de l'Intérieur un rapport sur «l'intégration économique des migrants et la valorisation de leur épargne » dans lequel il tentait d'évaluer les flux globaux d'argent et la part des sociétés de transfert dans ces envois. À une échelle plus réduite, l'argent de la diaspora commence à être convoité par différentes entreprises. En 2012 par exemple, une entreprise togolaise créait le site «228cado.com», sorte de supermarché en ligne où les émigrés togolais peuvent acheter des produits directement livrés au Togo à leur famille.

9. Cette pratique a été observée lors de chaque passage à l'aéroport. À Moroni par exemple, une émigrée d'une cinquantaine d'années s'arrêtait devant moi pour remplir sa fiche d'informations pour le visa et demandait à sa fille de reprendre le sac qu'elle avait oublié quelques mètres plus loin. En se tournant vers moi, elle dit en riant : «Y'a des millions là-dedans, 1500 d'une personne, 1000 d'une autre... » J'ai moi-même été souvent sollicité pour transporter des colis dans mes bagages.

10. Pour un point de vue critique sur ce marché, voir par exemple l'article de Sabrina Kassa [2008]. 
Si les flux du Sud vers le Nord sont invisibles, c'est avant tout parce qu'ils vont à rebours de la définition sociale et politique de l'émigré-immigré [Noiriel, 2007]. Dans les médias, les immigrés sont souvent décrits comme des individus qui, poussés par des écarts de développement importants, sont partis trouver à l'extérieur un emploi et une situation économique plus favorable. L'argent figure alors naturellement au centre de leurs préoccupations, puisqu'ils seraient venus en grande partie pour cette raison. La migration étant présentée comme une stratégie négociée au sein de la famille, qui vise à aider ses proches grâce à l'expérience migratoire, l'envoi d'argent est une constituante et un objectif de ce projet collectif [Boyer, 2005]. Cette vision réductrice des déterminants de la migration internationale est parfois reprise dans certains travaux sociologiques ${ }^{11}$.

Lors d'un entretien réalisé avec deux membres togolais d'une ONG qui travaille sur les questions de développement local, notamment sur des projets destinés à éviter le départ de jeunes togolaises, ceux-ci expliquèrent que la migration était un phénomène social ancien dans le pays, qui avait toujours eu pour fonction d'aider la famille. Pour les générations précédentes, qui partaient dans les pays voisins travailler dans des exploitations de café ou de cacao comme pour les plus jeunes, qui partent désormais dans l'ensemble de la sous-région, en Europe ou en Amérique du Nord, le départ avait pour but «de réaliser des biens au Togo », «d'envoyer de l'argent pour éduquer les petits frères ou pour construire la maison ». Ils précisèrent que pour «chaque famille où il y a un émigré, la famille a tendance à avoir un changement social ». Autrement dit, l'argent gagné est envoyé dans le pays d'origine pour participer au bien-être familial.

Cette aide financière peut avoir plusieurs usages : permettre à la famille de se nourrir convenablement, de se soigner, de travailler dans un petit commerce familial ou de construire une maison. Les envois d'argent depuis le pays d'accueil se donnent donc à voir concrètement dans le pays de départ, en transformant le paysage urbain et l'activité économique, notamment.

Plus encore, le fait d'envoyer de l'argent pourrait être une preuve de l'attachement des émigrés à leur famille et à leur pays. Il devient la matérialisation concrète d'un lien social et affectif. Lorsque je demandai à Muhamadi O. ${ }^{12}$, émigré comorien parti en France en 1983, comment il faisait pour garder le contact avec ses parents et ses proches restés aux Comores, il hésita puis répondit : «Pour garder le contact avec la famille, déjà, c'est les aider, communiquer. » Lors des dernières rencontres entre acteurs étatiques et acteurs de la coopération française et internationale aux Comores, les transferts d'argent de la diaspora ont été abordés sous cet angle de l'attachement dont ils sont le témoignage. Plusieurs acteurs interrogés se sont inquiétés, publiquement et en entretien, du fait que les deuxième et troisième générations de Comoriens nés en France, ont « une autre façon d'agir et de réagir »,

11. C'est notamment le cas dans les travaux de Jacques Barou ou d'Hugues Lagrange, ou plus largement dans les nombreux travaux de sociologie ou d'anthropologie des migrations qui expliquent l'émigration par la théorie des facteurs d'attraction et de répulsion (push/pull factors).

12. Les prénoms des personnes enquêtées ont été modifiés. 
qu'ils n'ont «plus l'amour du pays comme leurs parents » et que, par conséquent, « les transferts, ça va s'arrêter, mais vraiment net ». Envoyer de l'argent à distance, c'est en quelque sorte démontrer son attachement intact et faire preuve de sa capacité à être toujours là, au moins symboliquement, malgré les distances. En novembre 2012, lors d'une table ronde sur l'impact économique des migrations, Kadhy Sakho Niang, la secrétaire générale du Forum des organisations de solidarité internationale (FORIM) expliquait que le modèle subsaharien du « migrant qui aide la famille au pays est une espèce en voie de disparition» puisque certains jeunes quittent désormais leur pays en coupant les liens avec leur famille.

Le phénomène social de transfert monétaire, que l'on observe assez facilement lors des enquêtes de terrain, est repris et amplifié par différents acteurs économiques. Western Union est devenue spécialiste des campagnes publicitaires qui jouent sur ce rôle social de l'émigré. À la fin de l'année 2009, l'entreprise lançait une vaste campagne sur la plupart des sites d'information africains en ligne où l'on voyait sur l'image publicitaire un jeune Africain souriant, vêtu à l'européenne et où l'on pouvait lire : «Puis-je faire la fierté de ma famille ? OUI ! »

Mais ce sont avant tout les acteurs politiques chargés des migrations qui renforcent cette image. Ainsi, le 30 mars 2011, la Banque mondiale publiait un communiqué intitulé «Pour les émigrés africains, partir pour mieux contribuer» dans lequel on pouvait lire cette histoire :

« Il y a une vingtaine d'années, Yanga Dijiba prenait l'envol à partir de l'aéroport international de Ndjili à Kinshasa, capitale de la République démocratique du Congo. Destination : les États-Unis, où il continue de résider sans avoir jamais remis les pieds dans son pays natal. Aujourd'hui âgé d'une quarantaine d'années, il se souvient très bien des circonstances de son départ. "Issu d'une famille qui compte une dizaine d'enfants, j'avais bien vite réalisé qu'il nous faudrait gagner beaucoup d'argent. Or l'argent a tendance à suivre ceux qui ont une éducation solide. Â partir de là, j' ai compris ce qu'il me restait à faire", relate-t-il. » [Banque mondiale, 2011b]

En France, les envois de fonds sont un critère important pour définir, hiérarchiser et opposer les diasporas entre elles. En voulant définir la diaspora comorienne et ses spécificités, le consul de France aux Comores m'expliquait par exemple que les Comoriens sont « une population qui envoie beaucoup d'argent, en proportion de la population » et que l'on observe des «mouvements de fonds énormes ». L'argent envoyé depuis l'extérieur vers les Comores semblait être un trait majeur pour caractériser la diaspora comorienne et pour rendre compte d'un fort sens de la «solidarité » en migration. Le chef du projet du Programme de co-développement avec l'Union des Comores m'expliquait également que l'une des priorités de ce partenariat était d'aider à canaliser les fonds envoyés par la diaspora. Là encore, c'est l'immigré comme pourvoyeur de ressources et de projets pour son pays d'origine qui était valorisé.

Les pays d'émigration participent eux aussi à la définition politique du rôle de l'émigré. En effet, c'est en grande partie le constat de flux monétaires importants 
qui a, petit à petit, contribué à placer la question des migrations internationales sur l'agenda politique. Que ce soit au Togo ou aux Comores, la diaspora ne faisait pas réellement partie des priorités politiques. Aucun des deux pays ne disposait d'une structure dédiée aux émigrés comme ce peut être le cas au Mali ou au Sénégal. Mais devant le constat chiffré dressé par les organisations internationales, le sujet s'est imposé. Le nouveau commissaire chargé de la diaspora comorienne nommé en 2012, m'expliquait que «les bienfaits de la diaspora sont reconnus » par tous sans que rien ne soit mis en place formellement. D'après lui, les Comoriens constituent «l'une des diasporas qui pensent sérieusement au pays d'origine, à tel point qu'elle s'est substituée à l'État », jusqu'à devenir le premier «bailleur de fonds » du pays. Il faut donc désormais participer à l'organisation, la structuration et l'encouragement de ces activités diasporiques. Au Togo, un chargé de mission auprès du PNUD, qui coordonnait en 2010 un programme mené en partenariat avec le gouvernement, n'hésitait pas à surévaluer l'importance des transferts de fonds en indiquant que malgré les mauvaises relations passées entre le pouvoir politique et une diaspora considérée comme « une intelligentsia d'opposants », on pouvait être « sûr que la diaspora contribue vraiment au PIB » puisque le pays avait pu survivre, grâce à ces fonds envoyés, aux quinze années durant lesquelles il ne bénéficiait plus de l'appui de la coopération internationale. Désormais engagé dans un processus de formalisation de sa politique migratoire, le Togo a envoyé fin novembre 2012 à Genève une délégation à la $101^{\mathrm{e}}$ session du Conseil de l'Organisation internationale pour les migrations (OIM). À cette occasion, le représentant de la délégation l'avouait lui-même :

«Les migrants togolais regorgent de nombreuses ressources, notamment [...] d'importants moyens économiques et financiers qui pourraient être mobilisés pour faire face aux défis de développement du pays. Il n'y a donc point de doute aujourd'hui que la diaspora togolaise reste la première source de financement non génératrice de dette au pays. [...] Les transferts de fonds des migrants togolais représentent 9,4\% du PIB cette année. Ces fonds ont un impact réel sur la croissance économique, la réduction de la pauvreté et sur le renforcement du secteur financier. » ${ }^{13}$

Enfin, dans la presse togolaise et particulièrement dans la presse comorienne, les envois de fonds collectifs importants ou les activités associatives de la diaspora sont relayés, mettant en scène le rôle financier des émigrés.

L'objectif ici n'est pas de sous-estimer les transferts monétaires du Nord au Sud ${ }^{14}$. Les ethnographies menées auprès d'émigrés révèlent que l'envoi d'argent fait souvent partie de leurs obligations tacites [Laëthier, 2011]. En revanche, il

13. Certaines études ont pourtant montré qu'il fallait relativiser les effets positifs des apports financiers des migrants. Ils provoquent par exemple l'accroissement du prix des denrées alimentaires. Sur ces questions, voir les travaux de Flore Gubert [2002].

14. La revue économique mensuelle du Bureau national du Togo s'intéressait en juillet 2012 à la diaspora togolaise et écrivait : "Même si les deux tiers de la diaspora togolaise vit en Afrique, près des deux tiers des fonds transférés dans le pays proviennent plutôt de l'Occident. Ainsi, l'importance des transferts de fonds ne reflète pas la forte présence de la diaspora dans une localisation » [Banque Africaine de développement, Bureau national du Togo, 2012, p. 1]. 
apparaît que ce phénomène social mesurable, et largement mesuré, a été progressivement figé par les acteurs politiques, économiques et médiatiques à tel point qu'il n'est aujourd'hui plus questionné et qu'il est devenu un modèle naturel, une seconde nature du migrant, alors qu'il résulte en partie d'une vision réductrice des expériences migratoires. Plusieurs travaux de sciences sociales ont clairement expliqué que les migrants n'ont pas toujours vocation à devenir des développeurs [Gonin, 2005].

Pour autant, peut-on dire que toute circulation d'argent qui ne correspondrait pas à ce mouvement du Nord vers le Sud serait contre nature, qu'elle prendrait un caractère d'exceptionnalité ou ne serait pas digne d'intérêt ? Même si les transferts du Sud vers le Nord sont statistiquement moins présents ${ }^{15}$, faut-il pour autant abandonner leur étude ? En réalité, il est intéressant de chercher à mieux les connaître tant ils semblent pouvoir révéler d'informations sur les profils sociologiques des migrants et, par extension, sur leurs trajectoires migratoires.

\section{Du Sud au Nord, quand l'émigré reçoit de l'argent}

Plutôt que d'adopter une vision unidirectionnelle de la circulation monétaire, il faut garder à l'esprit que, bien souvent, les flux sont composites et s'entrecroisent sans que l'on puisse dire qu'une direction prenne le pas sur les autres. En effet, quel que soit le type de migration en jeu, l'argent circule fréquemment de manière multidirectionnelle.

\section{Des flux monétaires multidirectionnels}

Dans un même parcours migratoire, les flux monétaires peuvent prendre des directions diverses. Prenons l'exemple d'Antoine A., un jeune togolais venu étudier à l'université de Strasbourg en 2002 grâce à l'aide financière de son père qui vivait à Nantes et est aujourd'hui décédé. Après ses études d'économie et de gestion, il crée une entreprise d'import-export. L'acquisition de la nationalité française lui permet de multiplier plus facilement les va-et-vient entre la France, l'Allemagne, le Benelux et le Togo. En parallèle, il a fondé une association qui se propose d'aider les enfants togolais des zones rurales, ouvert à Lomé un « maquis » ${ }^{16}$ qui emploie sept personnes, acheté dans sa ville d'origine, Atakpamé, des terres pour lancer un futur projet agricole et acheté une maison à Lomé. Antoine passe le plus clair de son temps au Togo, et ses différents revenus générés à Strasbourg et à Lomé circulent dans plusieurs directions. Il prend en charge

15. Les rares auteurs qui s'intéressent aux envois de fonds du Sud au Nord semblent les évacuer très vite parce qu'ils seraient moins nombreux ou moins importants : «Les rares immigrés qui reçoivent des dons de leurs parents bénéficient d'un montant moyen assez élevé, si bien que ces dons s'apparentent davantage à une transmission partielle du patrimoine. Ils peuvent aussi bénéficier de prêts de leurs frères et sœurs, ce qui contribue à leur installation ici, mais les sommes d'argent qu'ils reçoivent sont bien moins élevées que celles qu'ils donnent. [...] Les immigrés sont dans l'ensemble des contributeurs nets face à leurs parents » [Attias-Donfut, Wolff, 2009, p. 260].

16. Terme désignant un bar-restaurant dans les pays d'Afrique francophone. 
financièrement sa femme et ses deux enfants, qui vivent en Belgique où il s'est un temps installé, ses deux petits frères qui étudient à Lomé, trois enfants qu'il scolarise à Atakpamé et cinq jeunes filles inscrites grâce à lui dans une formation de couture à Lomé. Avec des amis togolais vivant en France et en Allemagne, il organise tous les trois ou quatre mois une collecte de vêtements qui sont acheminés à Lomé par conteneur. Le cas d'Antoine illustre la difficulté à réduire un parcours migratoire à l'argent envoyé par un immigré à sa famille, tant dans ce cas comme dans bien d'autres, l'argent circule à la fois au Sud, au Nord, du Nord au Sud et du Sud au Nord.

Si l'on ne s'intéresse qu'à l'une des étapes des trajectoires migratoires, le départ, on perçoit également que les flux d'argent peuvent prendre des directions variées. Au moment de l'organisation d'un projet de départ, l'argent nécessaire à l'achat d'un billet d'avion, au paiement éventuel des frais de visa (qui impliquent également la production de justificatifs de ressources) et des frais d'hébergement peut provenir de différents lieux.

C'est d'abord la famille dans le pays d'origine qui peut financer le voyage. Ce fut le cas pour Wilfried Q. Ses parents, qui ont étudié et travaillé en France avant d'ouvrir une pharmacie à Lomé dans les années 1960, ont envoyé tous leurs enfants étudier hors du Togo. Le père de Wilfried en avait assez que son fils, alors jeune collégien, joue au « petit rasta révolutionnaire ». Il a alors financé son départ vers le Sénégal dans un établissement privé géré par les Frères maristes. En 1976, après des vacances passées en France chez ses sœurs, Wilfried pense y rester, mais son père l'envoie étudier le génie électrique au Nigeria. Après un coup d'État en 1983, son père accepte finalement de financer des études de mathématiques en France. Dans ce cas, l'argent circule principalement du Togo vers les pays d'accueil successifs simultanément. Mais les flux peuvent également partir du pays d'origine vers plusieurs destinations. Robert B., cadre dans une entreprise publique togolaise, m'a raconté que son père, avocat, et sa mère, inspectrice des douanes, avaient envoyé deux de leurs enfants étudier en France, deux autres aux États-Unis, un à Dakar et qu'il avait été pour sa part envoyé au Maroc. Ici, les flux partent tous du même lieu pour financer parallèlement des émigrations en Afrique, en Europe et en Amérique du Nord.

L'argent peut aussi provenir d'un autre pays du Sud. C'est grâce à l'argent d'un oncle inspecteur de police que Mohamed M. a pu quitter les Comores en 1952 pour le rejoindre à Diégo-Suarez, une ville du nord de Madagascar. À cette époque, Madagascar et les Comores sont encore françaises, il suffit donc à Mohamed d'un billet d'avion pour aller poursuivre sa scolarité chez son oncle, qui paiera à nouveau son voyage en 1964, pour qu'il aille travailler à Marseille, dans une compagnie maritime.

Les sommes nécessaires au départ d'un membre de la famille peuvent aussi être dépensées par quelqu'un qui a déjà émigré. Victor G., un jeune Togolais parti étudier à Paris en 2002 et revenu vivre à Lomé en 2005 après avoir été expulsé, explique que son père douanier ne pouvait pas payer les 9000 euros de frais 
d'inscription dans un BTS proposé par un établissement privé loméen, qui permettait d'aller étudier en France. Le projet d'études a donc été abandonné. C'est l'un de ses oncles vivant en France qui a relancé l'idée et l'a appuyé financièrement dans sa démarche d'inscription à l'université de Nanterre. Il lui a ouvert un compte bancaire, donné 1000 euros et payé le billet d'avion.

Enfin, l'argent peut parfois venir de plusieurs endroits à la fois. Lorsque Natumata C., une bachelière comorienne, a voulu s'inscrire dans une licence professionnelle de ressources humaines à Montpellier, il a fallu que plusieurs personnes participent à son projet. Son frère, qui avait étudié à Montpellier avant de travailler à la mairie de la ville, a préparé son inscription et a loué à son nom un appartement dont il était le garant. C'est son oncle, commerçant aux Comores, qui est devenu son tuteur et a apporté les garanties bancaires au consulat de France.

Les participations financières à la réalisation d'un projet migratoire sont donc multiples et l'argent peut provenir, exclusivement ou parallèlement, de proches qui travaillent dans le pays d'origine, dans un autre pays du Sud ou qui ont déjà émigré dans le pays d'accueil.

Résumer la circulation de l'argent en migration à un échange direct entre l'émigré au Nord et sa famille au Sud paraît donc réducteur. La circulation s'effectue à plusieurs échelles et intègre une dimension temporelle longue puisque les échanges, dons ou remboursements peuvent s'étaler sur plusieurs mois, années, voire générations. Difficile alors de décrire les envois de fonds du Nord au Sud comme la seule activité monétaire liée à la migration.

\section{Le sens de circulation de l'argent : un révélateur des profils sociologiques}

Au-delà de ces circulations croisées, il convient d'interroger ce que les directions prises par les flux monétaires en migration peuvent révéler. Il semble que le sens, en tant que direction et que signification, de circulation de l'argent dépend beaucoup des dispositions familiales et sociales du migrant. Le fait de pouvoir observer ou non cette pratique dans une trajectoire migratoire peut donc donner des indices sur le profil sociologique d'un migrant.

En effet, cette pratique est peu pensable dans le cas de familles qui appartiennent aux milieux sociaux les plus modestes ${ }^{17}$. Quand les membres d'une famille n'exercent aucune activité professionnelle ou que leur activité fournit peu de revenus (agriculture, pêche, élevage, petit commerce), le départ de l'un des leurs peut d'autant plus intimement être lié à la recherche de ressources financières. Même si les désirs, les objectifs et les intérêts à migrer ne se résument pas nécessairement à cette quête d'une rente migratoire, faire partir un membre de la famille constitue une opportunité économique. L'émigré est désigné comme celui dont le

17. L'application d'une classification en termes de classes sociales en Afrique a été longtemps débattue et reste une opération complexe [Balandier, 1965 ; Rivière, 1978]. Sans chercher à adopter une catégorisation rigide, nous donnons ici quelques traits généraux et saillants des caractéristiques sociales des individus enquêtés. 
rôle est de gagner de l'argent en migration et de le redistribuer aux siens. Il est un moyen d'accumulation de revenus pour la famille. Son départ doit à terme produire un retour sur investissement. D'autant plus si le départ a nécessité de consentir à des sacrifices financiers collectifs (cotisations dans des tontines, vente d'un terrain ou de bijoux, par exemple). Il est évident que l'envoi d'argent depuis le pays d'accueil constitue à la fois un but et une obligation plus ou moins tacite. Dans ce cas, l'envoi doit arriver le plus vite possible après l'installation de l'émigré devenu immigré. Abou O., est un fils d'agriculteurs. Aîné masculin d'une fratrie de neuf enfants, il a été choisi en 1989 pour partir en France. Il m'a expliqué que sa famille et lui avaient économisé pendant près de sept ans pour obtenir les 2000 à 3000 euros nécessaires à son départ. Arrivé d'abord à Marseille, il a finalement trouvé du travail à Nice au bout de trois mois grâce à des connaissances. Alors qu'il n'avait que 20 ans, il était devenu « comme le chef de famille » et il a ajouté : «Tout le monde comptait sur moi. » Alors que sa situation n'a été régularisée qu'en 1994, très vite, il a envoyé de l'argent à sa famille : «Quand j'ai gagné de l'argent, oui. Le deuxième mois, j'ai envoyé de l'argent ici. Parce que le deuxième mois y'avait le mariage de mon oncle, donc c'est le premier jour que j'ai commencé à envoyer de l'argent ici. »

Ce rôle est si fort que tous les émigrés issus de ce type de familles m'ont expliqué que leurs parents n'avaient pas eu besoin de leur rappeler, avant de partir, qu'il fallait qu'ils pensent aux leurs. L'obligation sociale est largement intériorisée. Le rôle familial est tacite, mais très prégnant. Il est d'ailleurs intéressant de noter que la famille restée au Sud ne demande pas beaucoup de précisions sur les modalités d'obtention de l'argent reçu. Abou résume très bien ce point, observé dans d'autres familles:

«Et bon, ma famille commençait à me demander comment j'ai réussi à avoir de l'argent, deux mois après. Mais bon, après ils s'en foutent. Dès qu'ils ont l'argent, c'est bon, ils sont tranquilles, ils cherchent pas à comprendre. »

Toutefois, l'envoi d'argent n'est pas une obligation permanente, même dans ces familles. D'abord parce que l'émigré, lorsqu'il a envoyé de l'argent régulièrement tout au long de son parcours, a fait ses preuves. En arrivant devant la maison familiale qu'il a financée, Abou m'a annoncé fièrement : «C'est ça mes 23 ans de là-bas!» Aujourd'hui, il ne paye plus systématiquement toutes les dépenses lorsqu'il vient en vacances et sa famille ne lui demande plus rien parce qu'il a scolarisé ses petits frères, soigné ses parents, construit la maison familiale et plus largement assuré la «tranquillité » familiale. Plus encore, il a réussi à remplir ce qui peut constituer un objectif de plus dans ces milieux sociaux, il a aidé son petit frère à venir en France et compte désormais sur lui pour prendre le relais.

« Ouais, j'ai construit tout seul et sans l'aide de personne. Et j'ai instruit mes petits frères et mes nièces qui ont fait les études à l'étranger. C'est depuis une dizaine d'années, tous les mois je les envoyais au moins, 100, 150 euros. Tous les mois. Ça me fait déjà dix ans que j'ai commencé ce travail et j'ai jamais raté un mois. Donc ça, si j'étais là, j'arriverais jamais à faire. Et maintenant, ils ont tous fini leurs études, ils ont tous commencé à travailler. Ça aide parce que maintenant moi, j'envoie rien à ma mère, ils s'en occupent », explique-t-il. 
Toutefois, cette obligation de donner à distance est beaucoup plus souple dès lors que l'un des membres de la famille a lui-même connu l'expérience de l'émigration avant de revenir s'installer dans son pays d'origine. C'est le cas d'Amin H., un Comorien de 66 ans qui est allé au lycée à Madagascar, a effectué son service militaire à la Réunion puis est parti en France en 1976, d'abord pour travailler dans une caserne militaire et enfin pour devenir gardien au port autonome de Marseille jusqu'à sa retraite en 2003. Il est alors revenu vivre aux Comores. Fils d'une famille de cultivateurs, il envoyait de l'argent à ses parents. Désormais, ce sont ses propres enfants qui complètent sa petite retraite. L'importance de cet argent ne fait aucun doute et il affirme clairement: «Ce sont mes enfants qui me financent, c'est là où ça me tient debout. » Quand je lui demande s'il aimerait que son fils vienne le retrouver, il répond : «Moi, si je dis à mon fils de rentrer, moi comment je vais faire ? Parce que j'attends qu'il m'envoie vingt euros. Et bah je dis qu'il faut qu'il reste là-bas ! » Tout au long de l'entretien, il a vanté les mérites de ses enfants qui lui permettaient d'acheter des médicaments, très chers aux Comores, ou qui l'avaient envoyé en pèlerinage à La Mecque. Cependant, puisqu'il connaît les difficultés financières que ses enfants affrontent à Marseille et qu'il a pu lui-même les expérimenter, ses attentes restent mesurées et il reste très compréhensif lorsque les envois d'argent se font attendre.

$\mathrm{Au}$ contraire, dans les familles urbaines socialement plus favorisées, dont la situation professionnelle permet d'obtenir des revenus conséquents (grands commerçants, chefs d'entreprise, cadres de sociétés publiques ou privées, etc.), qui ont un patrimoine élevé (propriété d'une ou plusieurs maisons, de terrains, de voitures), qui ont un capital scolaire important, ont parfois réalisé une partie de leurs études à l'étranger et qui jouissent d'un capital social important, envoyer de l'argent à ses parents ne fait pas sens. C'est ce qu'affirmait Rafik A., jeune Togolais venu étudier en France en 2002, et qui annonçait en riant, "J'envoie pas d'argent ! Mon père était préfet, il est à la retraite, ma mère fait du commerce, ils se débrouillent sans moi. »

Dans ces milieux sociaux, l'émigration ne vise pas la recherche de nouvelles ressources financières. Bien souvent, elle est présentée comme une nécessité, notamment pour poursuivre de bonnes études ou aller découvrir d'autres cultures, et devient un moyen de reproduire la situation sociale dominante de la famille dans le pays d'origine. Aux Comores, Kamila M., aînée d'une fratrie d'émigrés, qui est la seule restée à Moroni, insistait sur la différence qu'elle percevait entre des familles dans lesquelles l'argent constitue la première valeur ajoutée de la migration et d'autres, comme la sienne, dans lesquelles la migration a des retombées plus symboliques.

«Certaines familles, c'est vrai, c'est un plus financier. Parce que quand les gens partent travailler là-bas, ils envoient un plus. Surtout beaucoup plus des gens qui n'ont pas fait d'études, qui peuvent pas avoir un boulot ici, quand il va travailler là-bas, le boulot qu'il fait là-bas, quand il est éboueur, ou quand il est machin, mais c'est beaucoup. Il est mieux payé que s'il était resté, donc ça, c'est un plus. Parce qu'ici parfois y'a des familles qui vendent même des terrains pour envoyer leur 
enfant en France. [...] Moi mon père il travaillé à l'Organisation mondiale de la santé pendant longtemps donc lui c'était pas... Il attend pas un retour financier. Donc ceux qui sont là-bas ils travaillent, chacun s'occupe de lui-même. Même maintenant mon père, lui, il est toujours en activité. Il n'exerce plus la médecine ici. Il est à la retraite. Il est dans les conseils d'administration de certaines banques, donc financièrement il n'a pas besoin de l'apport de ses enfants. Il arrive quand même à se gérer lui-même donc. »

Elle a ponctué cette phrase par un rire puis, lorsque je lui demandai ce qui, pour elle, constituait un « plus » dans le fait d'avoir ses frères et sœurs à l'étranger, elle ajouta :

«Voilà moi c'est beaucoup plus, qu'est-ce que je dirais, beaucoup plus un plus culturel, un échange parce qu'on a souvent des échanges avec eux. Et puis je trouve qu'ils sont épanouis là-bas. »

Dans ces familles, le départ a été pris en charge par les parents et, une fois installé dans son pays d'accueil, c'est bien l'émigré qui continue à recevoir de l'argent. Dans les entretiens, ces envois étaient présentés comme de l'argent de poche. Cela a été le cas pour Ralf P., qui a quitté le Togo après son baccalauréat pour aller étudier à Dakar puis à Paris, où il habitait dans un logement acheté par ses parents. «J'ai pas quitté Lomé pour trouver l'eldorado [...] J'ai pas payé un franc pour le loyer [...] J'avais pas cette pression de réussite, cette obligation d'avoir une fortune... Tout ce que j'avais comme argent, j'ai fait le con avec sur Paris ! » Ce rapport beaucoup plus distancié avec l'argent rejoint tout à fait les propos tenus par Didier D. Fils d'un directeur d'une troupe de théâtre à Lomé, il est venu en France plusieurs fois, ainsi qu' au Canada, en Finlande ou en Espagne, pour différentes représentations de spectacles auxquels il participait. D'après lui, "C'était la belle vie, on nous payait en CFA, on ne connaissait pas la valeur là-bas [...] L'argent gagné, c'est avec les amis que tu dépenses ça, tu t'en fous, y'a la famille, c'est ça la belle vie. »

Les parents préfèrent envoyer de l'argent plutôt que de laisser leurs enfants à la charge d'autres membres de la famille, émigrés eux aussi. Lucien D. vivait à Lomé, chez sa mère qui travaillait comme cadre d'une compagnie aérienne. Pendant les vacances, il rejoignait son père, un homme d'affaires influent au Togo, qui vivait à Cannes. Un jour, son père a décidé qu'il allait mettre fin à son « exil » cannois, mais qu'il rentrerait sans Lucien, qu'il scolariserait dans un lycée français. Plutôt que de l'envoyer chez des cousins à Grenoble, il a préféré payer depuis le Togo un internat à Guingamp. Par la suite, grâce à l'argent envoyé par ses parents, Lucien a intégré une école de commerce à Nice avant de partir à Miami pour obtenir un Master of Business Administration à Florida Tech. Pour le père de Lucien, il s'agissait de montrer qu'il pouvait se passer d'un appui extérieur, qu'il n'avait pas besoin d'une quelconque entraide familiale ou solidarité communautaire et que ses revenus personnels lui permettaient largement de prendre son fils en charge, seul.

Enfin, si l'émigré issu d'une famille aisée n'a pas à envoyer d'argent à ses parents, il n'en est pas pour autant dégagé de toute obligation. L'argent qu'il reçoit de sa famille vivant au Sud implique un devoir de réussite, étudiante ou 
professionnelle. Lonlongo T. raconte comment ses deux parents, médecins à Lomé, l'ont ouvertement critiqué. Alors qu'ils lui avaient financièrement permis de passer son baccalauréat à Dakar, puis un BTS à Poitiers et surtout, d'intégrer une école d'ingénieur parisienne qui coûtait 6000 euros par an, il avait échoué dès la première année. Il concluait, souriant, en disant que «c'était cher payé ». Mais il expliquait surtout qu'il avait dû passer, difficilement, du statut d'étudiant au statut de travailleur et que ses parents ne l'avaient pas aidé dans cette période où il dormait de canapé en canapé, chez des amis. C'est également ce qui est arrivé à Kossi K., dont le père, avocat, était une figure politique togolaise de premier ordre. Il a été envoyé étudier le droit à Poitiers, en 1992. Kossi a complètement raté sa première année, à deux reprises. Son père a décidé de lui couper brutalement les vivres et a demandé à une cousine de lui payer le billet de retour à Lomé. En 2000, son père lui a obtenu un stage rémunéré comme steward dans une compagnie aérienne saoudienne. Le stage s'est mal passé et, après une altercation avec sa supérieure hiérarchique, on a demandé à Kossi de démissionner. Nouveau retour à Lomé. Kossi est désormais musicien, vit chez ses parents, mais a des relations très tendues avec eux.

\section{L'argent, un sujet inégalement abordé dans les entretiens}

Selon le sens de circulation des flux monétaires, de l'émigré vers sa famille ou de la famille vers l'émigré, l'envoi d'argent en migration peut être un révélateur à la fois du profil sociologique d'un émigré et des obligations familiales que son départ implique. Mais si la direction que prend l'argent en migration donne des indications précieuses, il est intéressant de noter que lors des entretiens, l'intérêt accordé aux questions d'argent est très variable.

Lors d'un entretien mené aux Comores, Adil M., fils de cultivateurs ruraux qui a quitté l'école très précocement, m'a expliqué dans les moindres détails comment sa famille avait préparé son départ en France. Ses proches avaient cherché dans tout le village un émigré prêt à échanger l'un de leurs terrains contre le paiement d'un billet d'avion et un court hébergement en France. Une fois l'argent obtenu, le grand frère d'Adil a opposé son veto au départ de son cadet et a préféré que les 2000 euros soient partagés au sein de la fratrie. L'argent étant au cœur de l'expérience d'Adil, nous avons parlé près d'une heure des implications techniques de son départ avorté en France.

Au contraire, dans d'autres cas, les questions financières, au même titre que les modalités administratives d'un départ sont un non-sujet, à peine abordé ou très vite évacué par des formules laconiques comme « avant de partir, j'ai fait le visa, le billet, tout ça ». L'économie domestique est largement commentée dans les milieux populaires, mais passée sous silence dans les milieux plus élitistes [Pinçon, Pinçon-Charlot, 1997]. C'est bien ce dernier point qui peut constituer un autre obstacle méthodologique à la connaissance approfondie des flux entre Sud et Nord. En effet, ceux qui envoient de l'argent depuis le Togo et les Comores ne sont pas ceux qui sont nécessairement les plus prompts à évoquer d'eux-mêmes ce sujet. 
Par ailleurs, la figure de l'émigré-immigré qui envoie de l'argent est si forte qu'elle agit comme un véritable poids et rend la parole plus difficile dans les familles aux situations économiques les plus précaires. Si, comme le précisait un chercheur comorien, le «Bon «Je viens ${ }^{18}$, c'est celui qui dégaine à tous les coups. Celui qui compte, on le critique », ou si, comme l'indiquait un journaliste togolais, les migrants valorisés sont ceux qui « font moins de bruit et aident la famille », chacun a intériorisé cette construction sociale et aller à l'encontre de ce modèle peut paraître honteux. Mais de la même manière, les entretiens ont montré comment la force d'un discours émergent qui lie migrations et développement peut pousser les individus des catégories sociales moyennes et supérieures à ne pas trop en dire, pour ne pas renforcer les décalages qui peuvent exister entre ce devoir de faire quelque chose pour son pays et leur trajectoire individuelle. Lorsque c'est l'émigré qui se trouve contraint de demander de l'argent aux siens suite à des difficultés financières, ce retournement peut être très mal vécu. Cela a été le cas pour Carole A. qui s'est retrouvée au chômage, en fin de droits, avec un fils à sa charge et a dû demander à son mari, qui était retourné préparer leur installation au Togo, de lui envoyer de l'argent alors même que le bilan annuel de son entreprise était mauvais : "C C'est lui qui envoyait de l'argent d'ici, c'est normal c'est lui le père de famille, c'est le sens du transfert que je trouvais aberrant [...] Et plus il envoyait de l'argent, moins il était prêt pour qu'on rentre, c'était un cercle vicieux. » Dès lors, il devient moins facile de dire, voire de confesser, quelque chose que l'on trouve anormal ou tout à fait contraire à ce qui semble devoir être.

Parler d'argent à un chercheur venu vous interroger, c'est à la fois répondre à une question que l'on considère comme intime et se confronter à des devoirs et obligations familiales et sociales. Raconter et mettre en mots la circulation de l'argent, c'est donc affronter un double tabou.

\section{Conclusion}

Au terme de cet article, on constate que si l'on ne considère pas l'argent des migrations comme une fin en soi, qui résout des problèmes de développement, mais plutôt comme une thématique de recherche qui peut éclairer les migrations internationales, la circulation monétaire pose peut-être plus de questions qu'elle n'apporte de réponses, pour le moment. Cependant, cette entrée permet d'observer que la circulation de l'argent en migration répond à des pratiques sociales très variées et à des situations très hétérogènes entre les familles et au sein des familles.

J'ai d'abord essayé de montrer comment l'argent des migrations s'est peu à peu imposé dans les arènes nationales et internationales, aussi bien au Nord qu'au Sud, comme une thématique centrale des migrations internationales. L'insistance sur les envois de fonds a surtout mis en avant de manière systématique la figure de l'immigré qui économise son maigre salaire pour contribuer au bien-être de sa

18. Terme utilisé aux Comores pour décrire les émigrés, les « Je viens de France ». 
famille dans son pays d'origine. Même si cette image ne semble correspondre qu'à une partie des migrants, elle est devenue la valeur étalon des migrations. Dès lors qu'envoyer de l'argent devient presque une composante intrinsèque de la condition d'émigré, les seuls flux monétaires observés sont ceux qui circulent du Nord au Sud. D'autres circulations multidirectionnelles, pourtant inhérentes aux migrations internationales, n'intéressent pas, ou très peu.

L'enquête menée à la fois en France, au Togo et aux Comores semble au contraire indiquer que l'argent circule dans tous les sens. Si les émigrés sont ceux qui envoient le plus d'argent, il apparaît qu'ils en reçoivent également. Si l'on regarde de plus près, se pencher sur le sens de la circulation monétaire en migration (sa direction), c'est observer le sens (la signification) que les familles des migrants donnent à l'argent et, par conséquent, obtenir des informations sur les migrants eux-mêmes. Le fait d'observer une circulation monétaire du Nord au Sud ou du Sud au Nord paraît pouvoir donner des indications sur les profils sociologiques des migrants.

Dans l'article, j'ai opposé deux idéaux types, celui des migrants issus de familles populaires, qui sont très largement incités à envoyer de l'argent à leur famille et celui des émigrés issus de familles élitistes, qui n'ont pas à envoyer d'argent, mais en reçoivent régulièrement tout au long de leur parcours. Entre ces deux pôles, le fait d'avoir fait des études, d'avoir vécu l'expérience de la migration ou de posséder un fort capital social, peut rendre possibles des situations intermédiaires dans lesquelles l'émigré peut envoyer moins d'argent, ou en recevoir à l'occasion. Il reste difficile d'affirmer mécaniquement : dis-moi qui tu es socialement, je te dirais comment l'argent circule dans ta famille ou au contraire, dis-moi quels sont les flux monétaires présents dans ta famille, je te dirais de quel milieu social tu viens. Toutefois, les tendances observées semblent bien refléter la polarisation décrite dans l'article.

Au-delà des indications sociologiques qu'il fournit, le sens de circulation des flux monétaires met en avant des éléments explicatifs intéressants des trajectoires migratoires. En effet, les obligations familiales et sociales plus ou moins pressantes peuvent différer ou accélérer des projets de retour, notamment. Il est par exemple difficile de rentrer définitivement si la famille considère que l'on doit encore envoyer de l'argent et que l'on n'a pas les moyens de compenser cette aide par l'obtention de revenus économiques au retour. À l'inverse, il est difficile de demander à ses parents les moyens de rester un peu plus en migration s'ils ont largement financé des études destinées à obtenir une situation professionnelle valorisante et assurée au retour.

Dans les discours institutionnels qui lient migrations et développement, il est demandé aux immigrés qui sont des travailleurs précaires de participer au développement de leur pays d'origine en envoyant de l'argent à distance. Quant aux étudiants ou travailleurs qualifiés, les pays d'accueil souhaitent qu'ils participent au développement en effectuant de courtes missions lors de vacances alors que les pays d'origine les invitent de plus en plus à effectuer des retours plus durables. 
Cet article a montré que ce n'est pas nécessairement le type de migration (migration de travail, étudiante, etc.) qui fait le sens de la circulation monétaire. C'est plutôt le profil sociologique de l'émigré qui va déterminer l'envoi ou la réception d'argent en migration.

Je rejoins donc ici l'appel lancé par Catherine Quiminal dans le livre cité au début de cet article [1991]. Elle y appelait à s'intéresser aux questions d'argent dans les différentes communautés auxquelles appartient l'immigré pour comprendre le fonctionnement même de ces communautés.

\section{Bibliographie}

AgENCE FRANÇAISE DE DÉVELOPPEMENT (AFD) [2012], «L'exemple des Comores », Série Transferts d'argent : la prédominance des voies informelles ?, 2 août : http://envoidargent.solidairesdumonde.org/archive/2012/07/27/serie-transferts-d-argent-la-predominance-des-voies -informel.html (page consultée le 2 avril 2013).

Attias-Donfut C., WolfF F.-C. [2009], Le Destin des enfants d'immigrés. Un désenchaînement des générations, Paris, Stock, 315 p.

Balandier G. [1965], «Problématique des classes sociales en Afrique noire », Cahiers internationaux de sociologie, vol. 38, p. 131-142.

BANQUe AFRICAINE DE DÉVELOPPEMENT (AfDB) [2008], Les Transferts des fonds des migrants, un enjeu de développement, Tunis, Banque africaine de développement, $84 \mathrm{p}$.

BANQue AFricaine DE DÉVElopPeMENT, BUREAU NATIONAL DU TOGO (BAD - TGFO) [2012], «Diaspora togolaise», Revue Économique mensuelle: http://www.afdb.org/ fileadmin/uploads/afdb/Documents/Project-and-Operations/Revue\%20\%C3\%A9conomique \%20TGFO\%20N\%C2\%B0\%201\%20juillet\%20\%2030\%20juin\%202012.pdf

BANQUE MONDIALE [2011a], « Les migrations, une chance pour l'Afrique », Newsletter, $\mathrm{n}^{\circ} 316$, 31 mars-6 avril 2011 : http://web.worldbank.org/WBSITE/EXTERNAL/ACCUEILEXTN/ NEWSFRENCH/0,,contentMDK :22874982 pagePK :64257043 piPK:437376 theSitePK: 1074931,00.html (page consultée le 13 mars 2013).

BANQUe MONDIALE [2011b], « Pour les émigrés africains, partir pour mieux contribuer », Nouvelles et évènements, http://go.worldbank.org/NAF5DH6FL0 (page consultée le 20 mars 2013).

BANQUE MONDIALE [2013], « Les migrants africains pourraient économiser 4 milliards de dollars par an sur les frais d'envoi de fonds », Reportage : http://sendmoneyafrica.worldbank.org/ fr/reportage (page consultée le 12 mars 2013).

Beaud S. [1996], "L'usage de l'entretien en sciences sociales. Plaidoyer pour l'“entretien ethnographique" », Politix, vol. 9, n 35, p. 226-257.

BOYeR F. [2005], «Le projet migratoire des migrants touaregs de la zone de Bankilaré : la pauvreté désavouée » in BILger V., Kraler A. (dir.), "African Migrations. Historical Perspectives and Contemporary Dynamics", Stichproben. Vienna Journal of African Studies, vol. 2, no 8 , p. 47-67.

BRÉANT H. [2012], « Démontrer le rôle positif des migrations internationales par les chiffres. Une analyse de la rhétorique institutionnelle du système des Nations unies », Mots. Les langages du politique, vol. $3, \mathrm{n}^{\circ} 100$, p. 153-171. 
CARbonnier G. [2010], «L'aide au développement une fois de plus sous le feu de la critique », Revue internationale de politique de développement, $\mathrm{n}^{\circ} 1, \mathrm{p} .141-147$.

COIFFARD M. [2012], « La coopération internationale sur les transferts de fonds des migrants, quels enjeux pour quelle perspective ? », Revue européenne des migrations internationales, vol. $28, \mathrm{n}^{\circ} 1$, p. 129-145.

DeDIEU J.-P. [2012], La Parole immigrée. Les migrants africains dans l'espace public en France (1960-1995), Paris, Klincksieck, 336 p.

De HaAs H. [2007], Remittances, Migration and Social Development, A Conceptual Review of the Literature, Genève, UNRISD, 38 p.

FILIPE C., Jỗo BABO M. [2013], «Émigration : en route pour le Mozambique », Jornal de negócios, Lisbonne, 12 mars, source: http://www.presseurop.eu/fr/content/article/ 3522551-en-route-pour-le-mozambique (page consultée le 7 avril 2013).

Freud C. [2009], De la Coopération française à la Banque mondiale. Mémoires du développement, Paris, Karthala, $256 \mathrm{p}$.

Garson J.-P., TAPINOS G. [1981], L'Argent des immigrés. Revenus, épargne et transferts de huit nationalités immigrées en France, Paris, INED, Presses universitaires de France, 352 p.

GAtinoIs C. [2012], «Le chômage pousse les Européens du Sud à l'exode», Le Monde, $1^{\mathrm{er}}$ novembre. Source : http://www.lemonde.fr/economie/article/2012/11/01/le-chomage-pousseles-europeens-du-sud-a-l-exode_1784382_3234.html (page consultée le 8 avril 2013).

Gonin P. [2005], «Jeux d'acteurs et enjeux territoriaux : quelles migrations pour quel développement ? L'exemple du bassin du fleuve Sénégal (République du Mali) », in CHAREF M., GonIN P. (dir.), Émigrés - immigrés dans le développement local, Agadir, Sud-Contact, $361 \mathrm{p}$.

GorCE B. [2008], «L'argent des immigrés va d'abord à leur famille », La Croix, 23 mai, source : http://www.la-croix.com/Actualite/Monde/L-argent-des-immigres-va-d-abord-a-leur-famille_NG_-2008-05-23-671596 (page consultée le 3 avril 2013).

Gubert F. [2002], "Do Migrants Insure Those who Stay Behind? Evidence from the Kayes Are (Western Mali)", Oxford Development Studies, vol. 30, n 3, p. 267-287.

GuRTNER B. [2007], «Un monde à l'envers : le Sud finance le Nord », Annuaire suisse de politique de développement, vol. $26, \mathrm{n}^{\circ} 2$, p. 57-80.

HiBou B. [1998], «Économie politique du discours de la Banque mondiale en Afrique subsaharienne. Du catéchisme économique au fait (et méfait) missionnaire », Les Cahiers $d u$ CERI, $\mathrm{n}^{\circ} 39$, Paris, Fondation nationale des sciences politiques, $46 \mathrm{p}$.

KAPUR D. [2004], "Remittances: The New Development Mantra?", G24 Discussion Paper Series, $\mathrm{n}^{\mathrm{o}}$ 29, Genève, United Nations Conference on Trade and Development, 22 p.

Kassa S. [2008], «Western Union, Money Gram. L'argent des migrants, miam, miam... », Regards, $\quad 1^{\text {er }}$ septembre: http://www.regards.fr/migrations/western-union-moneygram1-argent,3823 (page consultée le 5 avril 2013).

Klugman J. [2009], Rapport mondial sur le développement humain 2009. Lever les barrières : mobilité et développement humains, New York, PNUD, 237 p.

LAËTHIER M. [2011], Être migrant et Haïtien en Guyane, Paris, Comité des travaux historiques et scientifiques (CTHS), $319 \mathrm{p}$.

MARSAUd O. [2006], L'Europe s'intéresse à l'argent des immigrés, Radio France Internationale, 13 mars : Source : http://www.rfi.fr/actufr/articles/075/article_42428.asp (page consultée le 22 mars 2012). 
Mвoungou V. [2012], «Le transfert de fonds des migrants : les leaders de ce marché lucratif », Afrique Expansion Magazine, 19 avril : http://www.afriqueexpansion.com/le-transfert-defonds-des-migrants-/4043-le-transfert-de-fonds-des-migrants--les-leaders-de-ce-marchelucratif.html (page consultée le 15 mars 2013).

MinISTÈRE DES AfFAIRES ÉTRANGÈRES [2008], Les financements innovants pour le développement : http://www.diplomatie.gouv.fr/fr/politique-etrangere-de-la-france/aide-au-developpement-et/dispositifs-et-enjeux-de-l-aide-au/les-financements-innovants-pour-le/ (consultée le 2 mars 2013).

NoIRIEL G. [2007], Immigration, antisémitisme et racisme en France (XIX'-XXe siècles). Discours publics, humiliations privées, Paris, Fayard, 715 p.

Observatoire des pays d'Afrique, CARaïBes et Pacifique (ACP) SUR les migrations, MigraTION 4 Development [2012], Note d'information, «Promouvoir une attention accrue aux migrations et développement Sud-Sud, 24 juillet : http://www.migration4development.org/ sites/m4d.emakina-eu.net/files/tmp/note_dinformation_discussion_en_ligne_migrations_ sud-sud_0.pdf (page consultée le 13 mars 2013).

Pinçon M., Pinçon-Charlot M. [1997], Voyage en grande bourgeoisie. Journal d'enquête, Paris, Presses Universitaires de France, 184 p.

Quiminal C. [1991], Gens d'ici, gens d'ailleurs. Migrations Soninké et transformations villageoises, Paris, Christian Bourgois, 223 p.

RAtHA D. [2011], Leveraging Migration for Africa. Remittances, Skills, and Investments, Washington, Banque mondiale, Banque africaine de développement, $193 \mathrm{p}$.

Rivière C. [1978], Classes et stratifications sociales en Afrique. Le cas guinéen, Paris, Presses Universitaires de France, 296 p.

SAYAD A. [1999], «Les coûts et les profits de l'immigration », p. 118-124, in SAYAD A., La Double Absence. Des illusions de l'émigré aux souffrances de l'immigré, Paris, Le Seuil, $437 \mathrm{p}$.

Schмiтz J. (dir.) [2008], Migrants ouest-africains : miséreux, aventuriers ou notables?, Paris, Karthala, Politique africaine, $\mathrm{n}^{\circ}$ 109, $208 \mathrm{p}$.

Système D’obSERVATION PERMANENTE DES MigRATIONS (SOPEMI) [2006], Perspectives des migrations internationales, Paris, Éditions de l'OCDE, 340 p. 